Seasonal assessment of threats against

conservation of Inia geoffrensis humboldltiana

(Cetartiodactyla Iniidae) middle river basin Meta, Colombia

\title{
Valoración estacional de las amenazas contra la conservación de Inia geoffrensis humboldltiana (Cetartiodactyla Iniidae) en la cuenca del Río Meta, Colombia
}

\author{
Federico Mosquera-Guerra ${ }^{1 *}$, Carlos Parra ${ }^{2}$, Fernando Trujillo ${ }^{3}$, Alex Mauricio Jiménez-Ortega ${ }^{4}$ y \\ Hugo Mantilla-Meluk ${ }^{5}$
}

\begin{abstract}
'Fundación Omacha. Calle 84 No. 21 - 64 Barrio El Polo, Bogotá, Colombia. Email:federico.mosqueraguerra@gmail.com. ${ }^{2}$ Cormacarena. Cra 35 No 25-57 Barrio San Benito,Villavicencio, Colombia. Email: carlosparra@cormacarena.gov.co. ${ }^{3}$ Fundación Omacha. Calle 84 No. 21 - 64 Barrio El Polo, Bogotá, Colombia. Email: fernando@omacha.org. ${ }^{4}$ Grupo de investigación en Manejo de Fauna Silvestre Chocoana. Programa de Biología, Universidad Tecnológica del Chocó. Carrera 22 No 18 B-10 -Barrio Nicolás Medrano, Quibdó, Colombia. Email: alexmauriciojimenez@gmail.com. 5Programa de Biología, Universidad del Quindío. Carrera 15 Calle 12 Norte, Armenia, Colombia. Email: hugo.mantillameluk@gmail. com.

${ }^{*}$ Corresponding author
\end{abstract}

Introduction: As a consequence of population isolation by the presence of geomorphological barriers, dolphins from northern South America are subdivided into three species: I. geoffrensis (Amazon and Orinoco Rivers), I. boliviensis (Madeira River in Bolivia), and the recently described I. araguaiaensis inhabiting the hydrologic complex of the Araguaia -Tocantins Rivers in the northeastern Brazilian Amazon. The importance of pink dolphins at the ecosystems they inhabit is supported by: 1 ) its position as top predators in the food chain; 2) bioindicators for ecosystem health; 3 ) its cultural and mythological meaning for the local ethnic groups, such as Sikuanis from the Orinoco, and Ticuna for the Amazon; and 4) their economic value, mainly as a touristic attraction. The conservation status of Orinoquian populations of river dolphins (i. g. humboldtiana), has not been fully established. However, we know their populations are threatened by a series of human activities on both aquatic and terrestrial environments, affecting their occurrence across the basin. This research is part of an inter-institutional effort conducted between the Corporación para el Desarrollo Sostenible del Área de Manejo Especial de la Macarena (CORMACARENA) and Fundación Omacha, to monitor pink dolphin populations inhabiting the rivers: Meta, Manacacías, Yucao, and Cusiana, in order to identify conservation tensors affecting the species in the study area.

Methods: We conducted a multitemporal study to determine anthropogenic threats affecting dolphin populations during two hydrologic periods along $317.2 \mathrm{~km}^{-1}$ across the middle Meta River basin. We calculated the degradation index for streams of continental waters and density of the river dolphins, developed by Gómez-Salazar et al. (2012). Water quality, habitat transformation, species exploitation, and the increment in number of human settlements were identified as the main conservation tensors in the analyzed area.

Results: We obtained a high value in the index of degradation, with a potential increment in value through time. River confluences were identified as priority areas for the conservation of the species. The development of infrastructure projects on the main streams was identified as a future threat on freshwater ecosystems in the basin. Differences in hydrologic periods were identified as an important variable, not only affecting the ecological dynamic of the species, but also determining the anthropogenic tensors through time. Water quality, habitat modification, and species exploitation were particularly intense at low water level periods, while boat traffic, and fishing activities are more frequent at periods of high water levels in the region.

Discussion and conclusions: For the Meta River, water quality and species exploitation appear to be the major tensors for dolphins and they may explain the low densities recorded for the area in comparison with densities 
reported for the Amazon basin. Even though, we consider this analysis is not definite and more samplings are necessary to confirm this hypothesis. Finally, river confluences were identified as conservation hot spots for dolphin populations. We point out on the importance of river dolphins as umbrella species, ideal to develop conservation strategies in the region.

Key words: Degradation index, Meta River, threats and conservation, Tonina.

\section{Introducción}

El proceso de especiación del género Inia (familia Iniidae) fue resultado del aislamiento entre cuencas por procesos geomorfológicos, lo que dio lugar a tres especies: 1) I. geoffrensis, en los ríos Amazonas y Orinoco; 2) I. boliviensis, en la cuenca alta de río Madeira en Bolivia (la recientemente descrita), y 3) I. araguaiaensis, en el complejo hidrográfico formado por los ríos AraguaiaTocantins, en la amazonia brasilera (Trujillo y Diazgranados 2012; Hrbek et al. 2014).

La importancia de estos cetáceos en los ecosistemas acuáticos dulceacuícolas donde habitan se sustenta, por un lado, en su valor biológico como depredadores tope e indicadores de la salud del ecosistema; otro aspecto, es su significancia cultural y mitológica en etnias como los Sikuanis del Orinoco y los Ticuna del Amazonas; por último, en su valor económico, principalmente como atractivo turístico. El delfín rosado presenta una amplia distribución en el continente Sudamericano, ya que habita en los cauces principales, afluentes, lagunas e incluso en las áreas de inundación de las cuencas de los ríos Amazonas, Meta y Orinoco en Colombia (Da Silva 1994; Trujillo et al. 2013). Sin embargo, su estado de conservación actual es poco conocido, y sus poblaciones presentan una serie de amenazas causadas por actividades antrópicas desarrolladas en los ecosistemas terrestres y acuáticos. Estas amenazas, identificadas en las últimas dos décadas para las cuencas de los ríos Amazonas y Orinoco, han sido clasificadas en categorías que incluyen 1) biomagnificación en las redes tróficas de metales pesados como el mercurio; 2) contaminación por pesticidas y diferentes tipos de sustancias tóxicas empleadas en el mantenimiento de las extensas plantaciones de arroz, palma y procesamiento ilegal de alcaloides; 3) modificación de su hábitat por la implementación de proyectos de infraestructura; 4) interacciones negativas con las pesquerías industriales y artesanales, que implican capturas ilegales para usarse como cebo en la pesca de Calophysus macropterus (mota, simí o piracatinga) en la Amazonía y Orinoquia; 5) aumento en los niveles de ruido por el incremento en el tránsito de embarcaciones, y 6) alteraciones en los ciclos estacionales debido al cambio climático, lo que convierte a esta especie carismática en una especie fuertemente amenazada en el ámbito mundial (Ashley 1987; Trujillo et al. 2013).

\section{Material y métodos}

Durante marzo y mayo de 2014, se realizaron dos muestreos en los periodos de aguas bajas (marzo) y en ascenso (mayo; Figura 1 y Tabla 1). El trayecto comprendió muestreos desde las localidades de Puerto Gaitán (ríos Manacacías, Yucao y Meta) en el departamento del Meta $\left(3^{\circ} 05^{\prime}\right.$ latitud Norte y $-71^{\circ} 05^{\prime}$ longitud Oeste) hasta la confluencia de los ríos Meta y Cusiana $\left(4^{\circ} 31^{\prime}\right.$ de latitud Norte, $-71^{\circ} 50^{\prime}$ de longitud Oeste).

Fase de Campo. Para identificar las amenazas de origen antrópico en los sistemas hídricos evaluados y estimar la población de los delfines de río, se empleó una embarcación de fibra de vidrio de cuatro metros de largo y dos de ancho, propulsada por un motor de $85 \mathrm{hp}$. A lo largo de los dos muestreos en diferentes periodos hidrológicos, se recorrieron $317.2 \mathrm{Km}^{-1}$ a una velocidad promedio de $10-13 \mathrm{Km} / \mathrm{hr}$. Se ubicaron dos posiciones de observación en la embarcación: una en la proa con dos observadores, y otra en la popa con al menos dos observadores. Las 
observaciones desde proa fueron independientes de las observaciones en popa, y las posiciones de los observadores en cada plataforma fueron rotadas en un lapso de dos horas. Todos los observadores tenían experiencia previa con estudios de delfines de río (Gómez-Salazar et al. 2011).

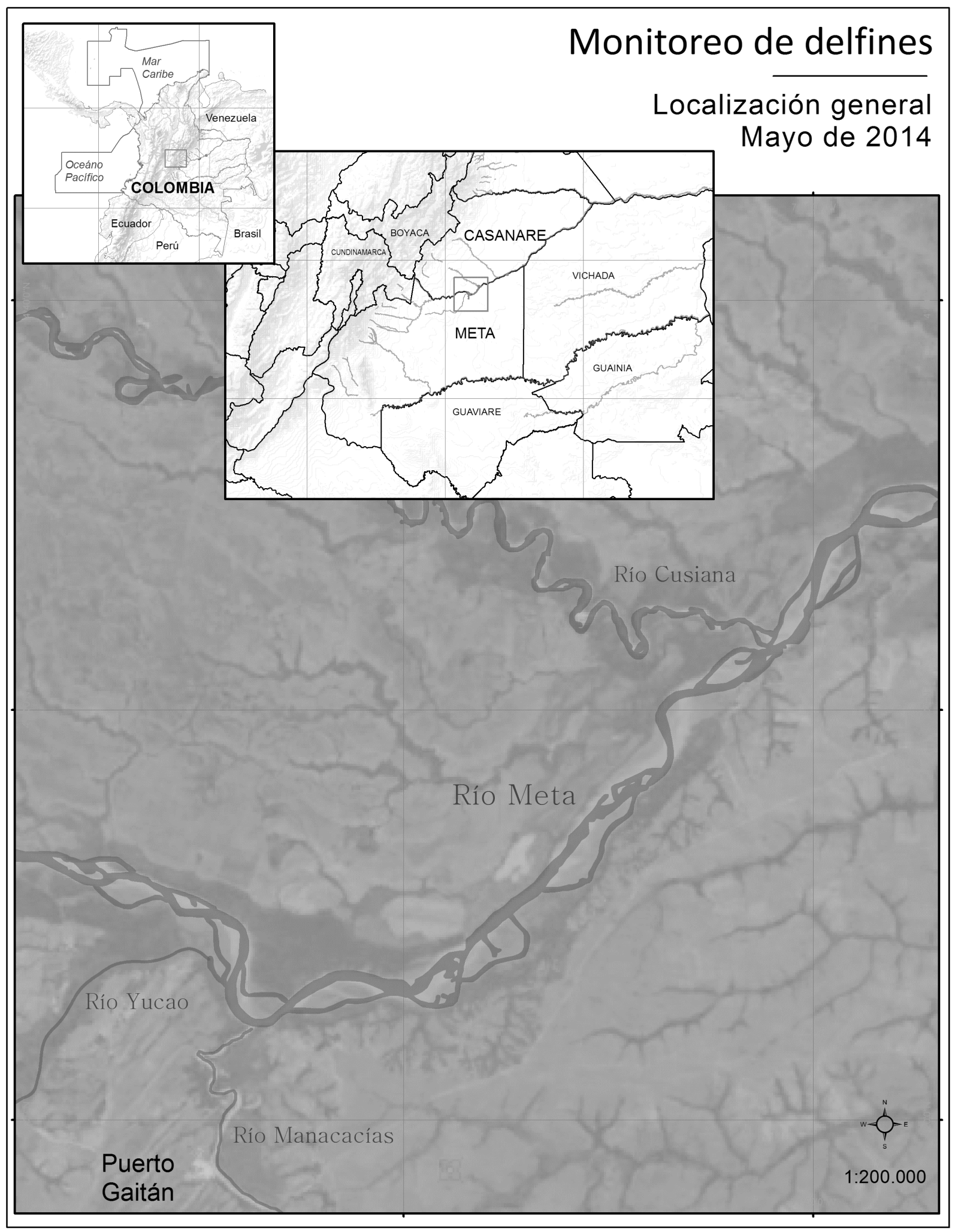

Figura 1. Ubicación espacial de las áreas de estudio en los ríos Manacacías, Yucao, Cusiana y Meta. 
Diseño de los transectos. Se establecieron cuatro tipos de hábitats con base en las conductas etológicas establecidas para los delfines de río y en criterios limnológicos para la cuenca, como a) río principal, b) confluencias, c) canales, y d) islas. El diseño de muestreo para los tipos de hábitat establecidos empleó una combinación de transectos en banda (paralelos a la orilla que mantenían una distancia de $150 \mathrm{~m}$ ) y transectos lineales (en un ángulo de $45^{\circ}$ respecto de la orilla; Gómez-Salazar et al. 2011). Los transectos en banda se realizaron en todos los tipos de hábitat y los transectos en línea solo se realizaron en el río principal. En el caso de los tipos de hábitat donde el ancho es menor a 400 m, se tomó la distancia a cada lado de la orilla. Se diligenció una planilla de esfuerzo de muestreo, donde se registraron los datos de distancia de los transectos, coordenadas de la zona y tipos de eventos (observaciones y amenazas de origen antrópico).

Tabla 1. Tipos de hábitat estudiados: área, longitud de los transectos y $N$ trans (número de transectos realizados por tipo de hábitat). $S$ transectos en banda de $200 \mathrm{~m}$ y $L$ transectos en línea.

\begin{tabular}{|c|c|c|c|c|c|c|c|c|}
\hline \multirow[t]{2}{*}{ Cuenca } & \multirow[t]{2}{*}{ Ríos } & \multirow[t]{2}{*}{ Tipo de Hábitat } & \multicolumn{2}{|c|}{$\begin{array}{l}\text { Área } \\
\left(\mathbf{k m}^{2}\right)\end{array}$} & \multicolumn{2}{|c|}{$\begin{array}{l}\text { Longitud } \\
\qquad(\mathrm{Km})\end{array}$} & \multicolumn{2}{|c|}{ Ntrans } \\
\hline & & & $\mathbf{S}$ & $\mathbf{L}$ & $\mathbf{S}$ & $\mathbf{L}$ & $\mathbf{S}$ & $\mathbf{L}$ \\
\hline \multirow{16}{*}{ Meta } & \multirow{4}{*}{ Manacacías } & Canal & 3.5 & & 35.0 & & 14.0 & \\
\hline & & Confluencia & 1.0 & & 10.0 & & 4.0 & \\
\hline & & Río principal & & & & & & \\
\hline & & Islas & & & & & & \\
\hline & \multirow{4}{*}{ Meta } & Canal & 8.8 & 1.6 & 40.0 & 7.2 & 16.0 & 9.0 \\
\hline & & Confluencia & 2.2 & & 10.0 & & 4.0 & \\
\hline & & Río principal & 23.6 & & 107.5 & & 43.0 & \\
\hline & & Islas & 6.0 & & 27.5 & & 11.0 & \\
\hline & \multirow{4}{*}{ Yucao } & Canal & & & 17.5 & & 7.0 & \\
\hline & & Confluencia & & & 10.0 & & 4.0 & \\
\hline & & Río principal & & & & & & \\
\hline & & Islas & 0.7 & & 17.5 & & 7.0 & \\
\hline & \multirow{4}{*}{ Cusiana } & Canal & 1.0 & & 25.0 & & 10.0 & \\
\hline & & Confluencia & 0.4 & & 10.0 & & 4.0 & \\
\hline & & Río principal & & & & & & \\
\hline & & Islas & & & & & & \\
\hline \multicolumn{9}{|l|}{ Total investigado } \\
\hline Todos los transectos & & & & & & 124.0 & 9.0 & \\
\hline
\end{tabular}

Tensores antrópicos y valoración del índice de degradación de ecosistemas de agua dulce. El índice de degradación para ecosistemas lóticos fue desarrollado por Gómez-Salazar et al. (2012) y considera diez tensores antrópicos (Tablas 2 y 3), agrupados en cuatro categorías: I) calidad del agua, II) modificación del hábitat, III) explotación de especies y IV) asentamientos humanos y ciudades. Cada tensor fue categorizado con valores de 0 a 3 de acuerdo con los cuatro tipos de impactos considerados: (0) cuando el disturbio es ausente/ no hay disturbio, (1) cuando el disturbio es bajo, (2) medio y (3) alto (Tabla 4). El valor del índice para las categorías (calidad del agua) es (I), modificación del hábitat; (II) y explotación de especies (III; Tabla 3). A continuación, se calculó el valor promedio de estos tensores para ser considerados en el valor general del índice, obtenido por medio de la sumatoria de los valores resultantes para las tres categorías evaluadas (Tabla 4). Se estableció el valor general del índice en el rango entre 0 y 8.5 (8.5 consiste en una alta degradación). La información relacionada con el tamaño de la población (asentamientos humanos 
Tabla 2. Principales tensores antrópicos responsables de la degradación de los sistemas acuáticos y sus impactos sobre los ecosistemas acuáticos. Fuente: Gómez-Salazar et al. (2012). Alcamo et al. (2005), Hoekstra et al. (2011), Revenga et al. (2000), Alkemade et al. (2009), Moyle and Randall (1998), Alcamo et al. (2003), GIWA (2002), Milà i Canals et al. (2009), Falkenmark (1997), Bennett et al. (2004), Karr y Chu (1999), Vörösmarty et al. (2000), Vörösmarty et al. (2010).

\begin{tabular}{|c|c|}
\hline Tensores antrópicos & Impactos de los tensores antrópicos en los ecosistemas acuáticos continentales \\
\hline Cantidad de agua & $\begin{array}{l}\text { Captación de agua para uso doméstico, industrial y necesidades agrícolas, reservorios con } \\
\text { capacidad de almacenamiento. }\end{array}$ \\
\hline Calidad de agua & $\begin{array}{l}\text { Fuente puntual y fuentes no puntuales de contaminantes (e. g., contaminantes orgánicos, } \\
\text { incremento de nutrientes, metales pesados, contaminación microbiana, compuestos tóxicos } \\
\text { orgánicos), partículas suspendidas, temperatura. }\end{array}$ \\
\hline Modificación del hábitat & $\begin{array}{l}\text { Caminos, represas, reservorios, transformación de la tierra, uso intenso de la tierra, agricultura, } \\
\text { vegetación removida, fragmentación. }\end{array}$ \\
\hline Explotación de especies & $\begin{array}{l}\text { Presión por pesquerías, prácticas destructivas de pesca (e.g., pesca con explosivos o } \\
\text { empleando sustancias tóxicas), capturas excesivas y descartes, acuicultura. }\end{array}$ \\
\hline Cambio climático & $\begin{array}{l}\text { Incremento en la temperatura del agua, disminución en la precipitación, incremento en la } \\
\text { acidificación, cambios en la producción primaria. }\end{array}$ \\
\hline Introducción de especies & $\begin{array}{l}\text { Incremento en las tasas de introducción de especies en los sistemas dulceacuícolas y éxito en } \\
\text { las tasas de esta introducción. }\end{array}$ \\
\hline
\end{tabular}

y ciudades, IV; Tabla 4). En el área de estudio en la cuenca media del río Meta se obtuvo usando bases de datos del censo de la población en las localidades asentadas sobre la cuenca del Meta (Tabla 4, DANE 2005). Las amenazas en un futuro de los ecosistemas dulceacuícolas continentales para cada tensor antrópico se establecieron mediante el conocimiento y las experiencias adquiridas a través del trabajo de los investigadores en la región, y por el acceso a información como el desarrollo de proyectos sobre recursos hídricos en la cuenca (Tabla 4).

\section{Estimación poblacional de los delfines de río en la cuenca media del río Meta}

Estimaciones de densidad y tamaño de grupo. Los datos obtenidos a través de los transectos en banda se estimaron empleando el software DISTANCE, versión 5.0 (Buckland et al. 2001; Thomas et al. 2002; Thomas et al. 2010; Gómez-Salazar et al. 2011). La densidad poblacional (D) se evaluó como:

$$
\mathrm{D}=\mathrm{nE}(\mathrm{i}) f(0) / 2 \mathrm{Lg}(0)
$$

donde n: Número de grupos observados; E (i) es el estimado del tamaño para cada grupo de la población en un hábitat tipo i; $f(0)$ es la observación de la densidad probable a distancia perpendicular 0; L es la longuitud del transecto total, y g (0) es la probabilidad de observación de un grupo en la línea de transecto.

Usando los datos de la plataforma de observación, se estimará el $g(0)$. La función de detección fue de aproximadamente $50 \mathrm{~m}$. El modelo de detección seleccionado fue Akaike'sInformationCreation (AIC; Burnham y Anderson, 2002).

Los valores de (Pk) estimados para la especie correspondieron a los calculados por Gómez-Salazar et al. (2011), para las cuencas del Orinoco-Amazonía de la especie, y correspondieron a P1 = 0.91 (Distancias del observador 5 - 100 y $100-150 \mathrm{~m}$ ) y P2 =0,523 (0 - 50 y $150-200 \mathrm{~m}$ ), de acuerdo con las áreas de detección del tipo de hábitat (Buckland et al. 2001). El $g_{(0)}$ fue tomado de Gómez-Salazar et al. (2011), siendo 0.947 (0.025) para Inia. 
Tamaño de la población: El tamaño de la población de delfines de río (Ni) para cada hábitat i fue calculado por $\mathrm{Ni}=\mathrm{A}_{\mathrm{i}} \mathrm{Di}$.

En general, el coeficiente de variación del total estimado en el río fue calculado de la siguiente manera:

$$
\mathrm{CV}\left(\mathrm{N}_{\mathrm{l}}\right)=\sqrt{\Sigma \mathrm{SE}}(\mathrm{Ni})^{2} / \Sigma \mathrm{Ni}
$$

Tabla 3. Tensores humanos y definiciones para cada categoría de impacto. Los códigos para valorar las categorías de impacto son (0) no disturbio registrado, (1) bajo disturbio, (2) disturbio medio, (3) disturbio alto. Algunos tensores antrópicos no incluyen categorías de impacto (-). Fuente: Gómez-Salazar et al. (2012).

Tensores antrópicos

I. Calidad del agua
Categorías de impacto

Baja (1)

Media (2)

Alta (3)
6. Hidrovías

\section{Explotación de especies}

7. En malles accidentales/ muerte de delfines por competición por recursos (peces)

\section{Matanzas deliberadas} de delfines de río para su utilización como carnada en la pesca de Calophyus macropterus (mota, simí o piracatinga)

9. Pesquerías

\author{
Número de delfines \\ muertos por enmalles / o \\ muerte directa \\ Número de delfines \\ muertos capturados para \\ carnada
}

\begin{abstract}
Cualquier tamaño
Estaciones turísticas

Cualquier tipo

Cualquier tamaño
\end{abstract}

Comercial, pesca artesan y deportiva, o transporte

Cualquier tamaño

100-200 km
Ro-100 km
Rutas esporádicas

$50-100 \mathrm{~km}$

Rango 50 km

Rango 50 km

Conocimiento de

rutas establecidas

$100-200 \mathrm{~km}$

$50-100 \mathrm{~km}$

Rango 50 km

$\begin{array}{ll}\text { 500-1000 km río } & 200-500 \text { km río } \\ \text { abajo o 100-200 km } & \begin{array}{l}\text { abajo o 50-100 km } \\ \text { río arriba }\end{array} \\ \text { río arriba }\end{array}$

500-1000 km río $\quad 500-1000$ km río abajo o 100-200 km río arriba

río arriba

$\begin{array}{ll}\begin{array}{l}\text { Raro (registrada al } \\ \text { menos dos veces en } \\ \text { el área) }\end{array} & \begin{array}{l}\text { Ocasional } \\ \text { (registrado } \\ \text { solamente una vez } \\ \text { por año) }\end{array} \\ \begin{array}{l}\text { Raro (registrada al } \\ \text { menos dos veces en } \\ \text { el área) }\end{array} & \begin{array}{l}\text { Ocasional } \\ \text { (registrado } \\ \text { solamente una vez } \\ \text { por año) }\end{array} \\ \text { Subsistencia } & \begin{array}{l}\text { Comercial, } \\ \text { destinación de las } \\ \text { ciudades dentro de } \\ \text { la cuenca del río }\end{array}\end{array}$

Rango 200 km Río abajo y/o dentro de $50 \mathrm{~km}$ río arriba

Dentro 200 km Río abajo y/o dentro 50 km río arriba

Frecuente (Registrado solamente una vez por mes)

Frecuente (e.g., pesquerías de mota establecida en el área)

Comercial, destinación de las ciudades dentro y fuera de la cuenca del río

IV. Ciudades y Asentamientos humanos

10. Tamaño de la población 


\section{Resultados y discusión}

Tensores antrópicos y valoración del índice de degradación de ecosistemas de agua dulce. Se calculó el valor general del índice de degradación de un ecosistema acuático continental considerando la propuesta de Gómez et al. (2012), cuyos rangos se encuentran entre 0 y 8.5 (8.5 se ubica en una alta degradación). Se obtuvieron valores altos, entre 6.2 y 6.5 , en los muestreos realizados, durante los meses de marzo (aguas bajas) y mayo (aguas altas) de 2014. Los resultados coinciden con lo expuesto por Gómez et al. (2012), quien reporta valores de 6.5 para el índice. Debe anotarse que la intensidad de estos tensores antrópicos presenta un comportamiento diferencial entre los dos periodos hidrológicos. Se incrementa su impacto durante el periodo de aguas altas en la categoría de explotación de especies, específicamente en la actividad de pesquerías. Lo anterior se debe al incremento de dicha actividad, estimulada por los procesos de migraciones reproductivas de la fauna íctica, generados a su vez por las precipitaciones, en las que se implementan artes de pesca, como redes y trasmallos, que aumentan la probabilidad de capturas incidentales de estos cetáceos. En relación con el periodo de aguas altas, se incrementan actividades como el tráfico de embarcación y la actividad pesquera (Tabla 5).

Tabla 4. Valor general del índice para medir cada impacto (alto, medio y bajo) y niveles de riesgo de amenazas de los ecosistemas acuáticos en degradación. El valor general del índice es la sumatoria del promedio para obtener las cuatro categorías de tensores antrópicos (calidad del agua, modificación del hábitat, explotación de especies, ciudades y asentamientos humanos). Fuente: Gómez-Salazar et al. (2012).

Valor general del índice

\begin{tabular}{|c|c|c|}
\hline Alto & \multicolumn{2}{|l|}{ Tensores antrópicos clasificados como altos o medios, no tensores } \\
\hline Medio & Tensores antrópicos clasificados como medios. & $\geq 3 y<4$ \\
\hline Bajo & Tensores antrópicos son clasificados como bajos o no conocidos. & $<3$ \\
\hline \multicolumn{3}{|c|}{ Riesgo de amenazas ambientales (en los próximos 10 años) } \\
\hline$\uparrow$ & \multicolumn{2}{|c|}{$\begin{array}{l}\text { Expectativa de la amenaza a incrementarse (e.g. planificación y construcción de proyectos en los ecosistemas } \\
\text { acuáticos, incremento en el número de estaciones, etc.). }\end{array}$} \\
\hline$\longrightarrow$ & \multicolumn{2}{|c|}{ Expectativa de un comportamiento similar, en algunos casos el incremento de la población es una expectativa. } \\
\hline$\downarrow$ & \multicolumn{2}{|c|}{ La expectativa del riesgo se reduce gracias algunas acciones de conservación en el área. } \\
\hline & El riesgo no registrado en el área y no presenta una expectativa de & ximos años. \\
\hline
\end{tabular}
tensores
Sumatoria de los diferentes tipos de

Se logró establecer en el área procesos de expansión de la actividad petrolera. Sumada a un modelo económico extractivo basado en el monocultivo de palma, soya y ganadería extensiva, se convierte en la principal amenaza contra la especie (Gómez-Salazar et al. 2012). Igualmente, se registraron vertimientos de aguas domésticas del municipio de Puerto Gaitán y una inadecuada disposición de los residuos sólidos debida a los asentamientos humanos ubicados en la ribera del río Meta y sus tributarios. Por otro lado, la actividad turística en esta zona genera gran cantidad de residuos sólidos, especialmente durante los festivales que se organizan en el municipio de Puerto Gaitán. Hay que añadir el aumento considerable de embarcaciones que circulan en zonas de importancia ecológica para la especie. 
Adicionalmente, el establecimiento de cultivos agroindustriales de las diferentes variedades de arroz (Oryza sativa), palma africana o aceitera (Elaeis guineensis) y soya (Glycine max) genera una importante transformación del paisaje y reduce la cobertura forestal nativa. Las actividades asociadas a estos modelos productivos, como el aprovechamiento forestal y la quema, terminan por eliminar la cobertura forestal de tipo arbóreo y arbustivo, y la reducen a plantas pioneras como las gramíneas, con menor capacidad de retener el suelo para evitar de esta forma su erosión y contaminación de fuentes hídricas por el aumento en los niveles de sólidos totales disueltos y suspendidos, así como por la conductividad y la turbiedad. Este fenómeno modifica en el corto plazo los ciclos biogeoquímicos y la productividad primaria en los sistemas acuáticos y altera la calidad físico-química y biológica del recurso hídrico.

Tabla 5. Valor del índice y riesgo de amenazas contra un ecosistema acuático en degradación, y tamaño de la población en la cuenca hidrográfica del río Meta. La dirección de las flechas indica si el riesgo de degradación es una expectativa que se incrementa, decrece o se mantiene. Valor general del índice es la sumatoria del promedio de las cuatro categorías de tensores antrópicos (calidad del agua, modificación de hábitat, explotación de especies, asentamientos humanos y ciudades. Abreviaturas: Muestreo 1 (realizado durante marzo, en el periodo de aguas bajas) y Muestreo 2 (realizado durante mayo, en el período de aguas altas). Fuente: GómezSalazar et al. (2012).

\begin{tabular}{|c|c|c|c|}
\hline Tensores antrópicos & $\begin{array}{l}\text { Gómez et al. } \\
\text { (2012) }\end{array}$ & Muestreo 1 & Muestreo 2 \\
\hline Valor general del índice & 6.5 & 6.2 & 6.5 \\
\hline I. Calidad del agua & 2.5 & 2.5 & 2.5 \\
\hline 1. Explotación petrolera & $3 \uparrow$ & $3 \uparrow$ & $3 \uparrow$ \\
\hline 2. Turismo & $2 \uparrow$ & $2 \uparrow$ & $2 \uparrow$ \\
\hline 3. Tráfico de embarcaciones & $2 \uparrow$ & $2 \uparrow$ & $2 \uparrow$ \\
\hline 4. Minería & $\mathbf{3} \uparrow$ & $\mathbf{3} \uparrow$ & $\mathbf{3} \uparrow$ \\
\hline II. Modificación del hábitat & - & - & - \\
\hline 5. Represas & - & - & - \\
\hline 6. Hidrovías & - & - & - \\
\hline III. Explotación de especies & $2 \rightarrow$ & $1.7 \rightarrow$ & $2 \rightarrow$ \\
\hline $\begin{array}{l}\text { 7. En malles accidentales/muerte de delfines por competición por } \\
\text { recursos (peces) }\end{array}$ & $2 \uparrow$ & $2 \uparrow$ & $2 \uparrow$ \\
\hline $\begin{array}{l}\text { 8. Matanzas deliberadas de delfines de río para su utilización } \\
\text { como carnada en la pesca de Calophyus macropterus (mota, simí o } \\
\text { piracatinga) }\end{array}$ & $1 \uparrow$ & $1 \uparrow$ & $1 \uparrow$ \\
\hline 9. Pesquerías & $3 \uparrow$ & $2 \uparrow$ & $3 \uparrow$ \\
\hline IV. Ciudades y asentamientos humanos & $2 \rightarrow$ & $2 \rightarrow$ & $2 \rightarrow$ \\
\hline 10. Tamaño de la población & 181,276 & 181,276 & 181,276 \\
\hline
\end{tabular}

Otra amenaza es el incremento en el tráfico de embarcaciones de transporte de pasajeros y mercancías que pueden ocasionar colisiones con los delfines y generar el desplazamiento de los lugares de caza y reproducción, como las confluencias, debido al aumento en los niveles de ruido. En las dos salidas de campo (marzo y mayo) se registraron 21 embarcaciones de transporte de los habitantes de la ribera del río y 16 de transporte de pasajeros en la ruta entre los municipios de Puerto Gaitán-Puerto Carreño (Figura 2). 
En cuanto a las posibles modificaciones de los hábitats en un futuro, existen planes para construir una hidrovía. Esto puede interferir con los patrones de inundación y causar la fragmentación de las poblaciones de delfines, así como la disminución del recurso íctico (WCD 2000). Cabe recalcar que la extinción del delfín de río Yangtzé o baiji (Lipotes vexillifer) en China se produjo principalmente como resultado de la fragmentación de sus poblaciones (Reeves et al. 2003).

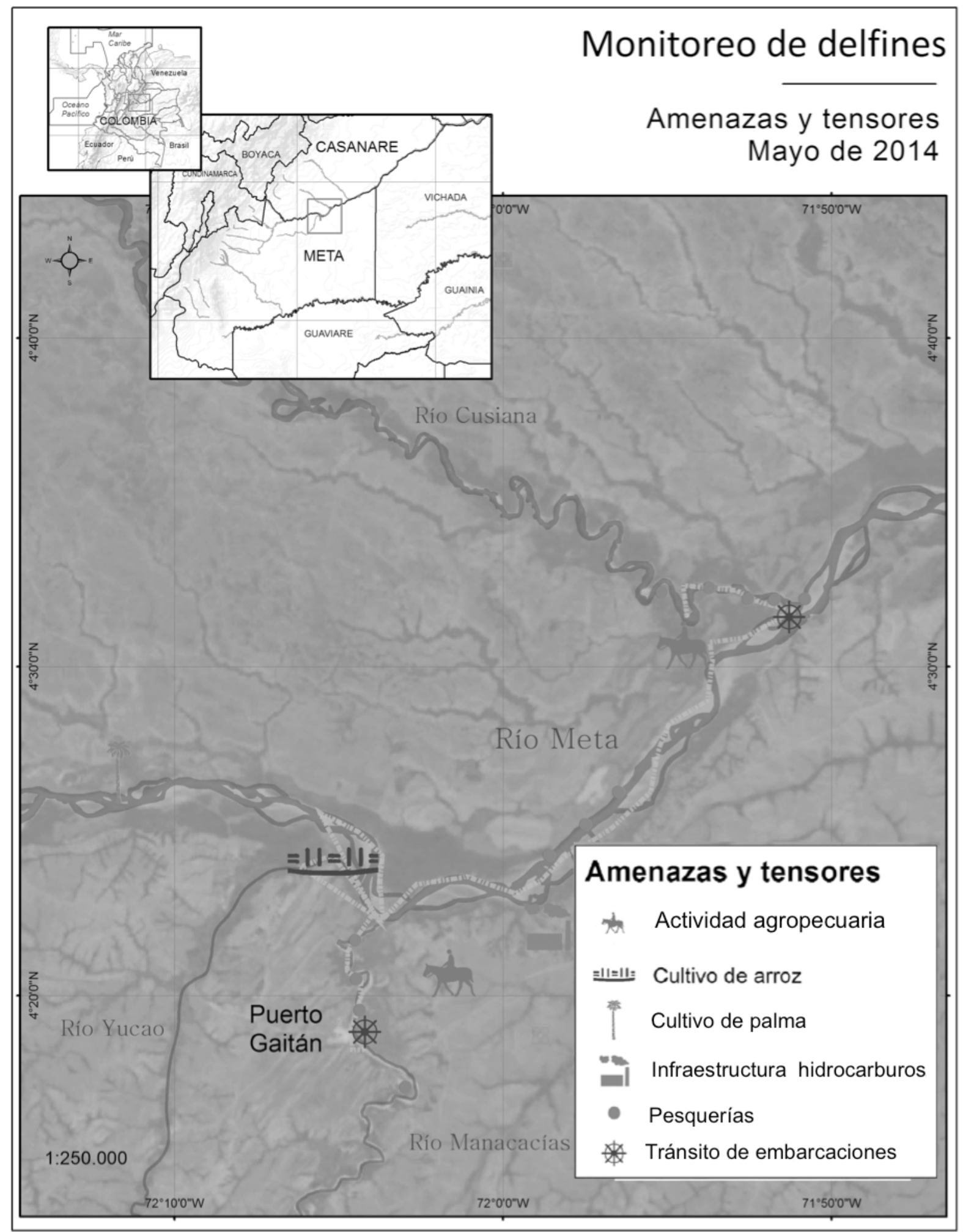

Figura 2. Ubicación espacial de las amenazas y tensores ambientales identificados en el área de estudio en los ríos Manacacías, Yucao, Cusiana y Meta. 
Actividades como la pesca deportiva y artesanal no solamente aumentan el tráfico de embarcaciones, niveles de ruido y peligro de enmallamiento accidental de estos cetáceos en las redes dispuestas a lo largo de los ríos asociados a la cuenca del río Meta. También vierten hidrocarburos y aceites en detrimento de la calidad física, química y biológica del agua (Figura 2). En total, se registraron 16 embarcaciones para esta actividad, de las cuales 11 correspondieron a actividades de pesca deportiva y 5 a labores de pesca artesanal desarrollada con artes de pesca perjudiciales para los delfines, como calandrios y trasmallos, que son estacionarios y están compuestos de redes de monofilamento. Lo anterior propicia que los delfines queden atrapados y puedan ahogarse. La actividad de desplazamiento a los lugares de pesca se realiza mediante las embarcaciones propulsadas por motor fuera de borda. De tal modo, no sólo aumentan las posibilidades de colisión y afectación a los delfines rosados, sino también al resto de la fauna asociada a los ecosistemas acuáticos. Esto se evidenció al encontrar a un individuo de babilla (Crocodylus sp.) muerto a causa de la colisión incidental con una embarcación.

Finalmente, el crecimiento demográfico de los núcleos o asentamientos urbanos se prevé que será considerable en los próximos años, debido principalmente a la migración de personas de diferentes lugares del país en busca de articulación con los diferentes modos actuales de producción regional, como la industria petrolera y la agroindustria.

Estimación poblacional de los delfines de río en la cuenca media del río Meta. Los resultados se presentan en función del número estimado de animales, entendido como la relación entre el total de individuos observados en cada tipo de hábitat por área $\left(\mathrm{Km}^{2}\right)$. Se obtuvo un número estimado de animales que osciló entre 22 individuos durante el periodo de aguas bajas (marzo) y 46 individuos en altas (mayo) en las 21 observaciones realizadas durante los dos periodos evaluados (Figura 3 y Tabla 6).

Tabla 6. Número estimado de delfines en cada uno de los muestreos realizados $\mathrm{Ni}$ (a) aguas bajas (marzo) y Ni (b) altas (mayo) en cada tipo de hábitat en la cuenca del río Meta.

\begin{tabular}{|c|c|c|c|c|c|}
\hline \multirow{2}{*}{ Cuenca } & \multirow{2}{*}{ Ríos } & \multirow{2}{*}{ Tipo de hábitat } & \multicolumn{3}{|c|}{$\begin{array}{l}\text { Número estimado de delfines } \\
\qquad \mathrm{Ni}=\mathrm{Ai}{ }^{*} \mathrm{Di}\end{array}$} \\
\hline & & & Área $\left(\mathbf{k m}^{2}\right)$ & $\mathrm{Ni}(\mathrm{a})$ & $\mathrm{Ni}(\mathrm{b})$ \\
\hline \multirow{16}{*}{ Meta } & \multirow{4}{*}{ Manacacías } & Canal & 3.5 & 0.1 & 0.4 \\
\hline & & Confluencia & 1.0 & 1.1 & 2.5 \\
\hline & & Río Principal & & & \\
\hline & & Islas & & & \\
\hline & \multirow{5}{*}{ Meta } & Canal & 10.4 & 0.3 & 1.0 \\
\hline & & Confluencia & 2.2 & 2.4 & 5.6 \\
\hline & & Río principal & 23.6 & 17.7 & 35.5 \\
\hline & & Islas & 6.0 & & \\
\hline & & Canal & & & \\
\hline & \multirow{2}{*}{ Yucao } & Confluencia & & & \\
\hline & & Río principal & & & \\
\hline & \multirow{5}{*}{ Cusiana } & Islas & 0.7 & & \\
\hline & & Canal & 1.0 & 0.03 & 0.1 \\
\hline & & Confluencia & 0.4 & 0.4 & 1.0 \\
\hline & & Río principal & & & \\
\hline & & Islas & & & \\
\hline Total investigado & & Todos los hábitats & 47.3 & 22.2 & 46.1 \\
\hline
\end{tabular}




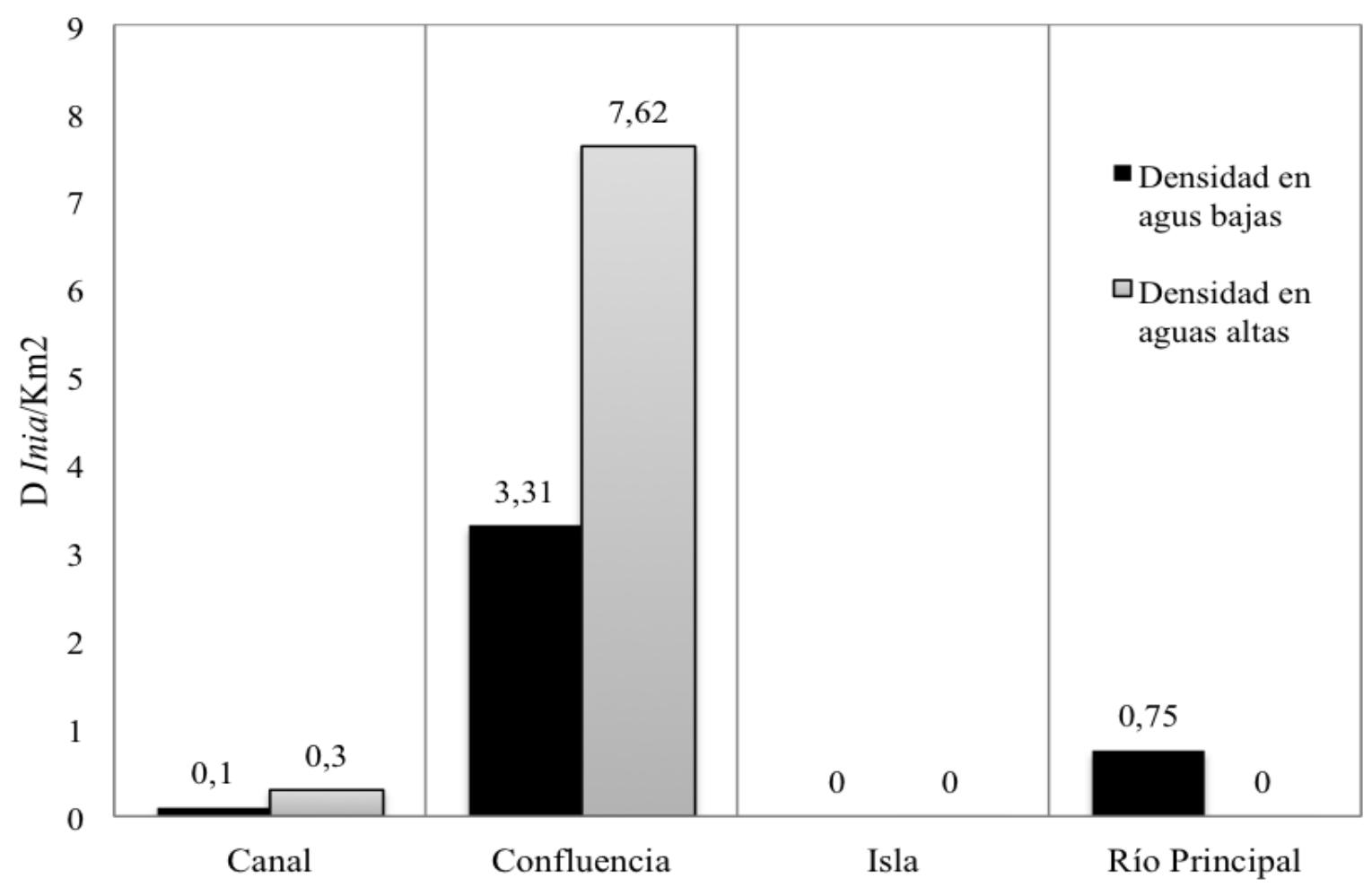

Tipos de Hábitat

Figura 3. Variación de la densidad de Inia geoffrensis humboldtiana en los diferentes tipos de hábitat muestreados durante las dos jornadas de campo realizadas en dos periodos hidrológicos.

La variación de las densidades de la especie durante los periodos de estudio se dio en función del tipo de hábitat: Confluencia D = $3.3-7.62$ Inia/ $\mathrm{Km}^{2}$, Río Principal $\mathrm{D}=0.75-1.5$ Inia $/ \mathrm{Km}^{2}$ y Canal D = $0.1-0.30$ Inia $/ \mathrm{Km}^{2}$. En el tipo de hábitat Isla, no se registraron observaciones (Figura 3 y Tabla 7).

El tamaño de grupo promedio para obtener los dos muestreos en aguas bajas y ascenso en el río Meta correspondió a 1.42 (0.74 SD) y estuvo influido por factores como el pulso de inundación que determina los niveles de productividad primaria de los sistemas acuáticos asociados a la cuenca del río Meta, al igual que la conectividad entre los tributarios como los ríos Manacías y Yucao, y el cauce principal del Meta en su cuenca media. De esta forma, se mejoró la conectividad y la funcionalidad de estos ecosistemas, así como las distribuciones de las especies que se encuentran asociadas a estas cuencas.

Tabla 7. Promedio de la tasa de avistamiento (n/l), estimativo de densidad (D) y coeficiente de variación para medir la tasa de encuentro CV (er) en el río Meta durante los periodos de aguas bajas y altas.

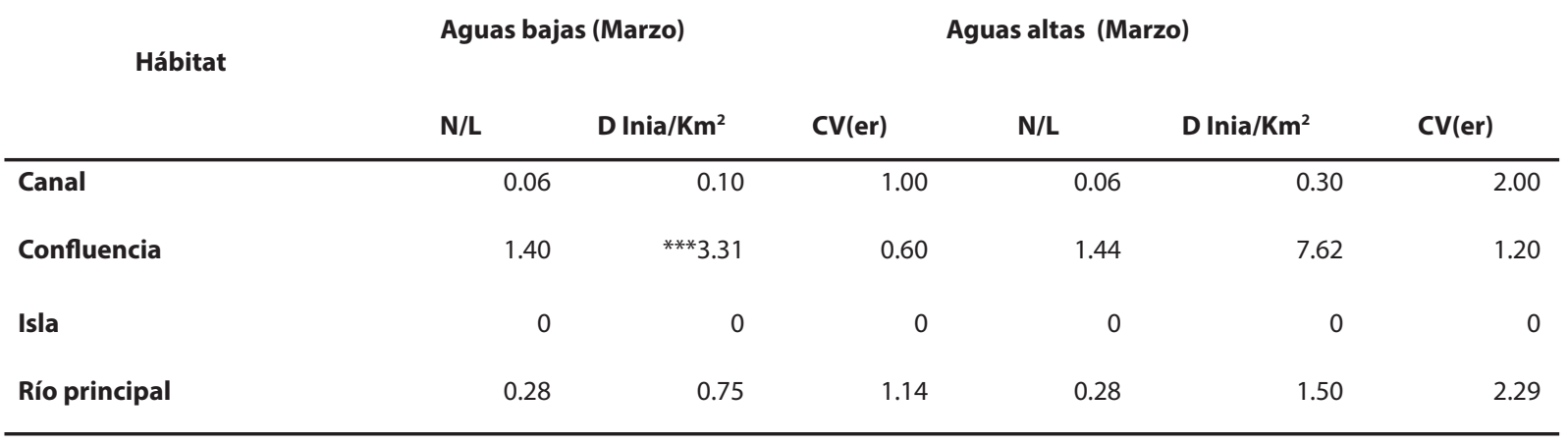


Los resultados durante el periodo de aguas altas coinciden con lo expuesto por GómezSalazar et al. (2011). Se reportaron densidades de $D=9.92 \mathrm{Inia} / \mathrm{Km}^{2}$ en el tipo de hábitat Confluencia, río Principal $\mathrm{D}=9.5$ Inia $/ \mathrm{Km}^{2}$ y Canal $\mathrm{D}=3.16$ Inia $/ \mathrm{Km}^{2}$. Sin embargo, GómezSalazar et al. (2011) registra densidades de $D=1.24$ Inia/ $\mathrm{Km}^{2}$ en el tipo de hábitat Isla. Esta diferencia de resultados reportados en el tipo de hábitat Isla posiblemente se deba a una mayor área muestreada (Puerto Gaitán-Puerto Carreño) por Gómez-Salazar et al. (2011) en segmentos más anchos del río.

Este estudio permitió identificar las confluencias entre los ríos Manacacías-Yucao, y Meta y Cusiana-Meta, como zonas de una alta productividad en relación con el recurso pesquero durante los diferentes periodos hidrológicos estudiados. Por lo tanto, se proponen como zonas prioritarias para la conservación de la especie, dada su importancia ecológica al presentar los mayores valores de densidades en los tipos de hábitat evaluados. Esto coincide
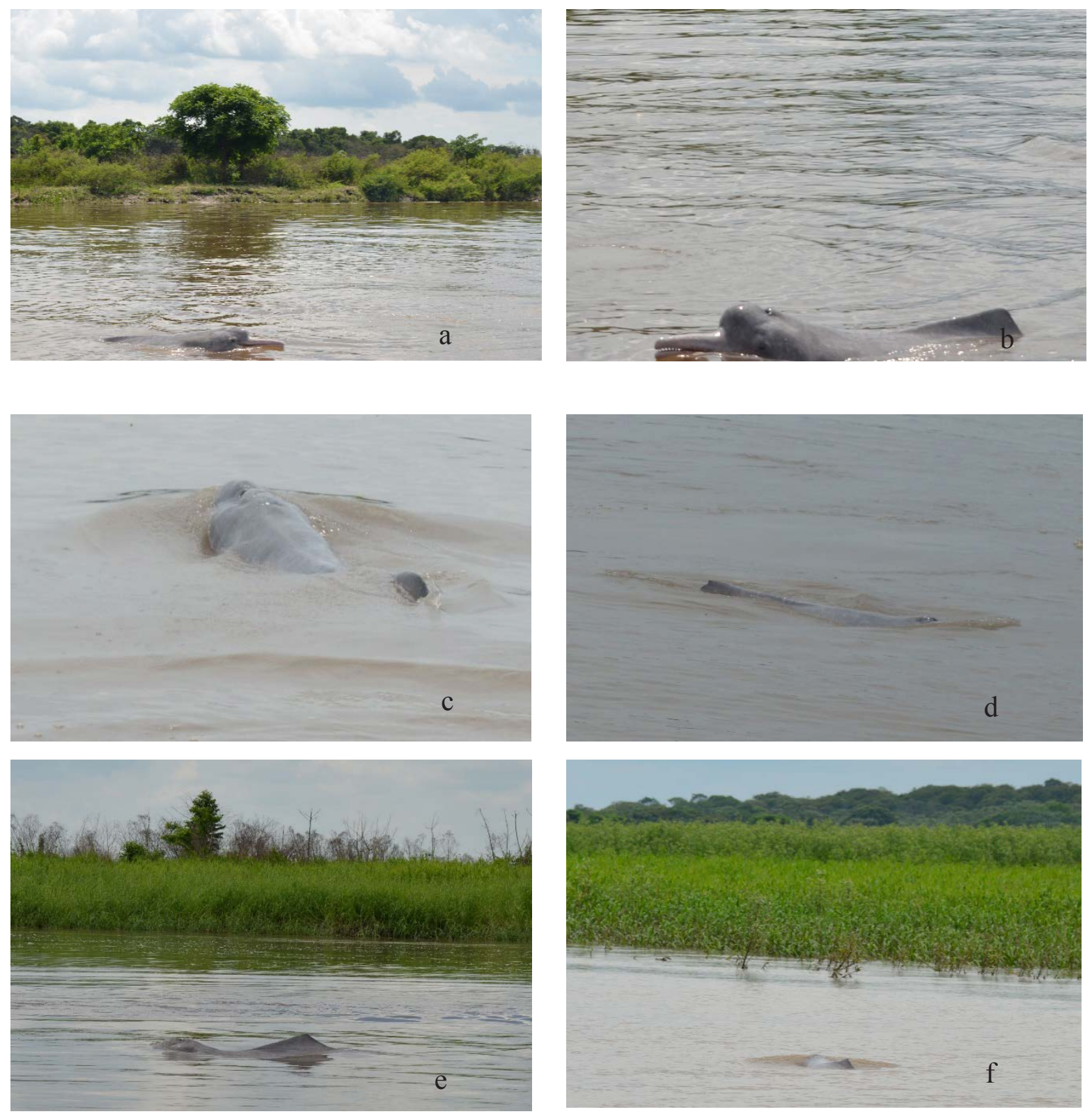

Figura 4. (a) y (b) Observaciones de individuos de Inia geoffrensis en río Yucao. (c) y (d) Individuos en tránsito por el río Meta. (e) Observación realizada en la confluencia de los ríos Meta, Yucao y Manacacías. (f) Individuo en la confluencia de los ríos Meta-Cusiana. 
con lo expuesto por Trujillo y Diazgranados (2004), y con Gómez-Salazar et al. (2011), quienes relacionan el mayor número de individuos en las confluencias en los río Meta y Orinoco (Figura 4). Posiblemente allí puedan presentarse conflictos con pesquerías por un recurso pesquero cada vez más escaso. La información obtenida permitió establecer la importancia de estas áreas como fuente de alimentación debido al encuentro de aguas y al paso obligado para la mayoría de los peces que realizan migraciones entre los ríos y sus tributarios. Estos lugares se convierten en algunos de los más preferidos por los delfines en sus actividades de alimentación (Smith, 1993; Trujillo, 2000; Trujillo et al. 2008). Asimismo, Trujillo y Diazgranados (2004) sugieren que haya esfuerzos de conservación en las confluencias. De esta forma, se garantizaría la presencia de los delfines y también de las especies que se encuentran asociadas a estos sistemas. Este estudio, junto con otros realizados
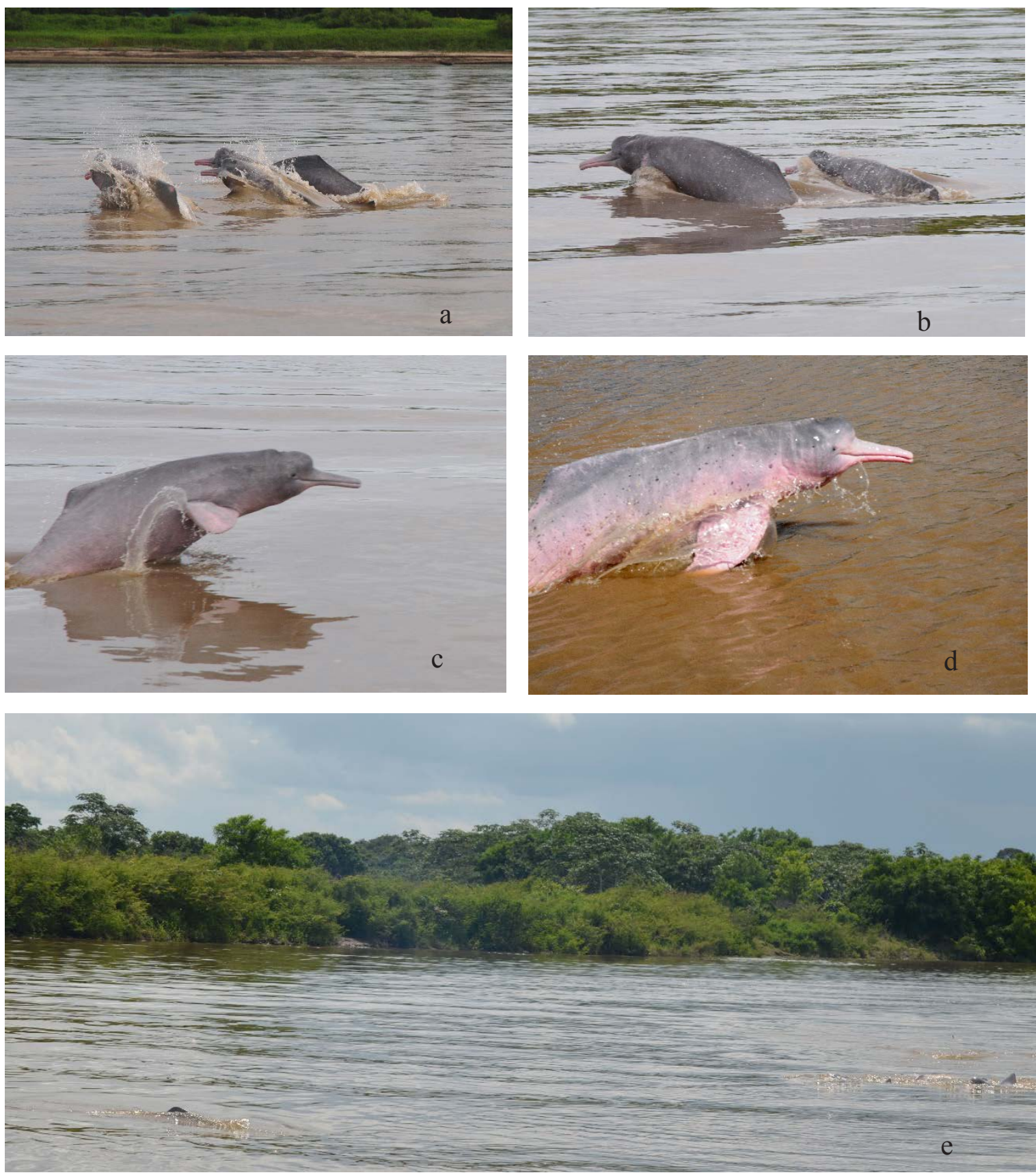

Figura 5. (a) y (b) Observaciones de dos individuos de Inia geoffrensis en río Yucao. (c) y (d) Individuos en tránsito por el río Meta. (e) Cinco individuos de la especie en la confluencia de los ríos Meta, Yucao y Manacacías. 
anteriormente, sugiere que las confluencias (ríos Meta-Manacacías y Yucao / Cusiana-Meta) constituyen los hábitats más importantespara I. geoffrensis en la cuenca del río Meta. En dichos lugares ocurren algunas actividades muy importantes para los delfines, como la alimentación, la socialización, la reproducción y la crianza (Figura 5).

La intensidad en estas alteraciones ambientales de origen antrópico, como la disminución en la cantidad y calidad del agua, la modificación del hábitat, la explotación de especies y el incremento en los asentamientos humanos (producto de los modelos productivos agroindustriales e hidrocarburos que se desarrollan actualmente en la cuenca del río Meta) podrían incidir en los resultados obtenidos. Podrían así presentarse bajas densidades de delfines en los diferentes tipos de hábitats en relación con otras áreas de estudio, como la Amazonía de Colombia. De esta manera, dichos mamíferos acuáticos se presentarían como indicadores del estado de salud de los ecosistemas acuáticos donde habitan (Gómez-Salazar et al. 2011).

\section{Conclusiones}

Los ecosistemas acuáticos evaluados en las áreas de estudio presentan un índice de degradación alto, influido principalmente por la alteración en la calidad del agua, la modificación del hábitat y la explotación de especies. Del mismo modo, se observa que el incremento de los asentamientos humanos en la región posiblemente se incrementen con el tiempo.

La intensidad en estas alteraciones ambientales de origen antrópico, así como su tendencia a incrementarse a lo largo del tiempo podrían reflejarse en bajas densidades de delfines en relación con otras áreas menos intervenidas, además de su posible disminución poblacional a largo plazo.

Algunas de las confluencias más importantes (puntos calientes: hot spots), identificadas como propias de los delfines de río durante la investigación, han sido establecidas como áreas prioritarias para conservar la biodiversidad en la cuenca. En consecuencia, los delfines constituyen una valiosa herramienta al ser empleados como especies sombrilla en las diferentes iniciativas de conservación o indicadores del estado de salud de los ecosistemas a una escala regional en la cuenca del río Meta.

Se sugiere fortalecer el proceso de educación ambiental en aspectos como buenas prácticas de pesca, uso de combustibles y motores fuera de borda con pescadores y motoristas igualmente en buenas prácticas de avistamientos y turismo responsable.

Finalmente consideramos importante establecer un diálogo con el sector productivo regional Hidrocarburos, agroindustria (porcícola, plantaciones de palma de aceite, soya y arroz) a fin de generar buenas prácticas agropecuarias y ambientales que permitan garantizar los atributos ecológicos y ambientales de la cuenca del río Meta.

\section{Agradecimientos}

Este proyecto se realizó como parte del proyecto Caracterización de las prácticas potencialmente conflictivas con los delfines de río y estimaciones de su abundancia en los ríos Meta, Manacacías y Yucao. Agradecemos el apoyo de la Corporación para el Desarrollo Sostenible del Área de Manejo Especial de la Macarena (CORMACARENA) y a , Whitley Fund for Nature-Fondation Segré y WWFColombia. De manera especial agradecemos a todas las personas de Puerto Gaitán por su ayuda durante el trabajo de campo. Finalmente agradecemos a los revisores anónimos que, con sus sugerencias y aportaciones, hicieron de este un mejor trabajo. 


\section{Resumen}

Introducción: El proceso de especiación del género Inia (familia Iniidae) fue resultado del aislamiento entre cuencas por procesos geomorfológicos, lo que dio lugar a tres especies: 1) I geoffrensis, en los ríos Amazonas y Orinoco; 2) I boliviensis, en la cuenca alta de río Madeira en Bolivia (la recientemente descrita), y 3) I araguaiaensis, en el complejo hidrográfico formado por los ríos Araguaia-Tocantins, en la amazonia brasilera. La importancia de los delfines rosados en los ecosistemas acuáticos dulceacuícolas donde habitan se sustenta, por: 1) Son los depredadores tope en las redes tróficas; 2) bioindicadores de la salud de los ecosistemas; 3 ) su significancia cultural y mitológica en etnias como los Sikuanis del Orinoco y los Ticuna del Amazonas; 4) Su valor económico, principalmente como atractivo turístico. El estatus de conservación de las poblaciones del delfín de río (I. g. humboldtiana), es poco conocido. Sin embargo, sus poblaciones se encuentran amenazadas por actividades humanas realizadas en los ambientes acuáticos y terrestres, afectando su distribución a lo largo de la cuenca. Esta investigación participa de un esfuerzo interinstitucional de la Corporación para el Desarrollo Sostenible del Área de Manejo Especial de la Macarena (CORMACARENA) y de la Fundación Omacha. EI objetivo fue evaluar, monitorear y generar estrategias para la conservación de las poblaciones del delfín rosado en la cuenca media del río Meta y en sus tributarios Manacacías, Yucao y Cusiana.

Materiales y Métodos: Se llevó a cabo un estudio multitemporal a fin de establecer las amenazas de origen antrópico y la densidad de la especie durante dos periodos hidrológicos a lo largo de $317.2 \mathrm{~km}^{-1}$ lineales en la cuenca media del río Meta y sus tributarios. Para estudiar los cursos de agua muestreados, se calculó el índice de degradación en corrientes de aguas continentales desarrollado por Gómez-Salazar et al. (2012). La alteración en la calidad del agua; modificación del hábitat; la explotación de especies, y el incremento en los asentamientos humanos fueron identificados como tensores para la conservación de la especie en el area de estudio.

Resultados: Se registró valores altos para el índice de degradación, con potencial incremento a futuro. Las confluencias son identificadas como áreas prioritarias para la conservación de la especie. El desarrollo de proyectos infraestructura fueron identificados a lo largo del tiempo como la principal amenaza a la conservación de los ecosistemas acuáticos asociados a esta cuenca. Los diferentes periodos hidrológicos fueron identificados como una variable importante, afectando la dinámica de las especies y determinando la intensidad de los tensores antrópicos durante los periodos hidrológicos evaluados. Calidad del agua, modificación de hábitat, y explotación de especies se presentaron durante el periodo de aguas bajas; tráfico de embarcaciones y actividades de pesca son más frecuente durante el periodo de aguas altas para la region.

Discusión y conslusiones: Para el río Meta, la calidad del agua y la explotación de especies fueron los mayores tensores para los delfines presentando bajas densidades para el área de estudio en comparación con las densidades reportadas para la cuenca del Amazonas. Se considera que este análisis no es definitivo y más estudios son necesarios para confirmar esta hipótesis. Finalmente, las confluencias de los ríos fueron identificadas como (puntos calientes: hot spots) para la conservación de los delfines. Los delfines constituyen una valiosa herramienta al ser empleados como especies sombrilla, ideal para el desarrollo de estrategias de conservación en la región.

Palabras clave: Amenazas y conservación, índice de degradación, río Meta, Tonina.

\section{Literatura citada}

Alcamo, J., P. Döll, T. Henrichs, F. Kaspar, B. Lehner, T. Rösch, y S. Siebert. 2003. Global estimates of water withdrawals and availability under current and future "business-as-usual" conditions. Hydrological Sciences Journal 48:339-348.

Alcamo, J., D. van Vuuren, W. Cramer, T. Masui, J. Alder, Y K. Schulze. 2005. Changes in ecosystem services and their drivers across the scenarios. In: Ecosystems and human well-being 2:297-373. Millenium Ecosystem Assessment. Washington, EE. UU.

Alkemade, R., M. van Oorschot, L. Miles, C. Nellemann, M. Bakkenes, y B. Ten Brink. 2009. GlOBiO3: a framework to investigate options for reducing global terrestrial biodiversity loss. Ecosystems 12:374-390.

Ashley, J. M. 1987. African Palm Oil: Impacts in Equador's Amazon. Cultural Survival Quarterly 11:55-60.

Bennett, H. H., M. Mullen, P. Stewart, J. Sawyer, y E. Cliff Webber. 2004. Development of an invertebrate community index for an Alabama coastal plain watershed. Journal American Water Resource Association 40:43-51.

Buckland, S. T., D. R. Anderson, K. P. Burnham, Y J. L. Laake. 2001. Introduction to Distance sampling: Estimating abundance of biological populations. Oxford University Press. Oxford, Reino Unido. 
Burnham, K. P., Y D. R. Anderson. 2002. Model selection and multimodel inference: A practical information-theoretic approach. Springer-Verlag. New York, EE. UU.

Departamento Administrativo Nacional de Estadística (DANE). 2005. Censo General 2005. http://www. dane.gov.co/index.php/poblacion-y-demografia/censos.

DA SıIva, V. M. F. 1994. Aspects of the biology of the Amazonian dolphins genus Inia and Sotalia fluviatilis. Cambridge University Press. Cambridge, Reino Unido.

Falkenmark, M. 1997. Meeting water requirements of an expanding world population. Philosophical Transactions of the Royal Society of London. Serie B: Biological Sciences 352:929-936. London, Reino Unido.

Global International Water Assessment (GIWA). 2002. Methodology. University of Kalmar, UNEP. Kalmar, Sweden.

Gómez-Salazar, C., F. Trujlllo, M. Portocarrero-Aya, y H. Whitehead. 2011. P opulation, density estimates, and conservation of river dolphins (Inia and Sotalia) in the Amazon and Orinoco river basins. Marine Mammal Science 28:124-153.

Gómez-Salazar, C., M. Coll, y H. Whitehead. 2012. River dolphins as indicators of ecosystem degradation in large tropical rivers. Ecological Indicators 23:19-26.

Hoekstra, A. Y., A. K. Chapagain, M. M. Aldaya, y M. M. Mekonnen. 2011. The Water Footprint Assessment Manual: Setting the Global Standard. Earthscan, Reino Unido.

Hrbek, T., V. M. F. da Silva, N. Dutra, W. Gravena, A. R. Martin, y I. P. Farias. 2014. A new species of river dolphin from Brazil or: how little do we know our biodiversity. PLoS ONE 9(1): e83623.

KaRR, J. R., Y E. W. ChU. 1999. Restoring Life in Running Waters: Better Biological Monitoring. Island Press. Washington, EE. UU.

Milà i Canals, L., J. Chenoweth, A. Chapagain, S. OrR, A. Antón, y R. Clift. 2009. Assessing freshwater use impacts in LCA: Part I - inventory modelling and characterisation factors for the main impact pathways. International Journal of Life Cycle Assessment 14:28-42.

Moyle, P. M., Y P. J. Randall. 1998. Evaluating the biotic integrity of watersheds in the Sierra Nevada, California. Conservation Biology 12:1318-1326.

Reeves, R. R., B. D. Smith, E. Crespo, y G. Notarbartolo di Sciara. 2003. Dolphins, Whales and Porpoises: 20022010 Conservation Action Plan for the World's Cetaceans. IUCN/SSC Cetacean Specialist Group, IUCN. Gland, Switzerland and Cambridge, Reino Unido.

Revenga, C., J. Brunner, N. Henninger, K. Kassem, y R. Payne. 2000. Pilot Analysis of Global Ecosystems: Freshwater Systems.

Sмітн, B. 1993. Status and conservation of the Ganges River dolphin Platanista gangetica in the Karnali River, Nepal. Biological Conservation 66:159-169.

Thomas, L., S. T. Buckland, K. P. Burnham, D. R. Aanderson, J. L. LaAke, D. R. Borchers, Y S. Strindberg. 2002. Distance sampling. Encyclopedia of environmetrics 1:544-552.

Thomas, L., S. T. Buckland, E. A. Rextad, J. L. Laake, S. Strindberg, S. Hedley, J. R. B. Bishop, T. Marques, y K. P. Burnham. 2010. Distance software: Design and analysis of distance sampling surveys for estimating population size. Journal of Applied Ecology 47:5-14.

Trusilzo, F. 2000. Habitat use and social behavior of freshwater dolphin Inia geoffrensis (de Blanville, 1817) in the Amazon and Orinoco basins. Ph.D. Thesis, Aberdeen University. Scotland, Reino Unido.

Trujillo, F., y M. C. Diazgranados. 2004. Variación estacional en el uso del hábitat del delfín de río, Inia geoffrensis humboldtiana, en la Orinoquía Colombiana. En: Fauna Acuática de la Orinoquía Colombiana (Diazgranados M. C., y F. Trujillo, eds.). Instituto de Estudios Ambientales para el Desarrollo. Pontificia Universidad Javeriana. Bogotá, Colombia.

Trujillo, F., M. Portocarrero, y C. Gómez. 2008. Plan de Manejo y Conservación de Especies Amenazadas en la Reserva de Biosfera El Tuparro: Delfines de río, Manatíes, Nutrias, Jaguares y Tortugas del 
género Podocnemis. Proyecto Pijiwi Orinoko (Fundación Omacha -Fundación Horizonte Verde) Forest Conservation Agreement. Bogotá, Colombia.

Trujlllo, F., y M. C. Diazgranados. 2012. Delfines de río. Embajadores de la conservación en la Amazonía y Orinoquía. Fundación Omacha-CEPCOLSA. Bogotá, Colombia.

Vörösmarty, C. J., P. Green, J. Salisbury, y R. B. Lammers. 2000. Global water resources: vulnerability from climate change and population growth. Science 289:284-288.

Vörösmarty, C. J., P. B. Mclntyre, M. O. Gessner, D. Dudgeon, A. Prusevich, P. Green, S. Glidden, S. E. Bunn, C. A. Suluivan, y C. R. LiermanN. 2010. Global threats to human water security and river biodiversity. Nature 467:555-561.

Summited: October 23, 2014

Review: April 2, 2015

Accepted: May 2, 2015

Associated editor: Juan Pablo Gallo 
388 THERYA Vol.6(2):371-388 